\title{
Impact of probiotics on pathogen survival in an innovative human plasma biofilm model (hpBIOM)
}

M. Besser ${ }^{1^{*}}\left(\mathbb{D}\right.$, J. Terberger $^{1}$, L. Weber ${ }^{1}$, B. Ghebremedhin' ${ }^{2}$, E. A. Naumova ${ }^{3}$, W. H. Arnold ${ }^{3}$ and E. K. Stuermer ${ }^{4}$

\begin{abstract}
Background: Despite of medical advances, the number of patients suffering on non-healing chronic wounds is still increasing. This fact is attended by physical and emotional distress and an economic load. The majority of chronic wounds are infected of harmful microbials in a protecting extracellular matrix. These biofilms inhibit wound healing. Biofilm-growing bacteria developed unique survival properties, which still challenge the appropriate wound therapy. The present in-vitro biofilm models are not suitable for translational research. By means of a novel in-vivo like human plasma biofilm model ( $\mathrm{hpBIOM}$ ), this study systematically analysed the influence of 3 probiotics on the survival of five clinically relevant pathogenic microorganisms.
\end{abstract}

Methods: Human plasma was used to produce the innovate biofilm. Pathogenic microorganisms were administered to the plasma. By stimulating the production of a fibrin scaffold, stable coagula-like discs with integrated pathogens were produced. The five clinically relevant pathogens P. aeruginosa, S. aureus, S. epidermidis, E. faecium and C. albicans were challenged to the probiotics L. plantarum, B. lactis and S. cerevisiae. The probiotics were administered on top of the biofilm and the survival was quantified after $4 \mathrm{~h}$ and $24 \mathrm{~h}$ of incubation. For statistics, two-way ANOVA with posthoc Tukey's HSD test was applied. P-value > 0.05 was considered to be significant.

Results: SEM micrographs depicted the pathogens on the surface of the fibrin scaffold, arranged in close proximity and produced the glycocalyx. The application of probiotics induced different growth-reducing capacities towards the pathogens. B. lactis and S. cerevisiae showed slight bacteria-reducing properties. The survival of C. albicans was not affected at all. The most antimicrobial activity was detected after the treatment with L. plantarum.

Conclusions: This study successfully reproduced a novel human biofilm model, which provides a human wound milieu and individual immune competence. The success of bacteriotherapy is dependent on the strain combination, the number of probiotics and the activity of the immune cells. The eradicating effect of L. plantarum on P. aeruginosa should be emphasized.

Keywords: Human plasma biofilm model, hpBIOM, Chronic wounds, Bacteriotherapy, Probiotics, L. plantarum, B. lactis, S. cerevisiae

\footnotetext{
*Correspondence: Manuela.Besser@uni-wh.de

${ }^{1}$ Institute of Translational Wound Research, Centre for Biomedical

Education and Research (ZBAF), Witten/Herdecke University, Stockumer

Street 10, 58453 Witten, Germany

Full list of author information is available at the end of the article
} 


\section{Background}

The demographic change is attended by an increased incidence of the development of chronic wounds leading to a significant socio-economic burden. If a wound displays no signs of healing after 4 weeks, despite of appropriate wound management, it is defined to be a chronic wound. Most patients with chronic wounds suffer from basic diseases that inhibit the perfusion of the tissue, e.g. diabetes, immobility or peripheral vascular insufficiencies. These wounds are from the beginning considered to become a chronic state [1].

The development and progressive maturation initiates with the adhesion and the attachment of bacteria and fungi preferentially on damaged tissue, followed by colonisation and infection via proliferation $[2,3]$. The surrounding milieu will be re-organized to a protecting extracellular polymeric substance (EPS) by the microorganisms. It is postulated, that $60-80 \%$ of non-healing wounds are challenged to these biofilms [4-6]. The dimension with regard to thickness and diameter varies from some micrometres to a few millimetres [7]. Persistent biofilms affect the well-orchestrated tissue repair process, leading to a prolonged inflammatory phase and, concomitantly, a delayed wound healing. Only $6 \%$ of acute wounds are affected by biofilms [4].

Bacteria in biofilms are at least tenfold more resistant to systemically as well as topically applied antibiotics than their planktonic variants, because of their improved survival mechanisms $[8,9]$. They show slower growth rate, are able to transfer genes mediating resistances to antibiotics and, due to the EPS, the penetration of antibiotics and other biocides is aggravated [10-12]. During wound therapy, patients are subjected to surgical interventions to destroy the biofilm matrix for cleansing of the wound area. Beside the painful procedure, tissue debridement consequently leads to an increased wound area but success often fails $[1,13]$. The most common strains in post-operative wound infections are Pseudomonas aeruginosa, Enterococcus faecium and Staphylococcus aureus [9]. The importance of finding new treatment methods for wound infections is underlined by the fact, that the recently published WHO recommendation list for R\&D of new antibiotics contains more than five wound associated pathogens. The investigated strains in this study are all ranked high or critical priority $[14,15]$.

In summary, wound healing is impaired by biofilms and successful strategies overcoming this challenge in the wound management are missing.

Alternative therapeutic approaches have to be assigned, one could be the bacteriotherapy. Bacteriotherapy involves the application of non-pathogenic microorganisms (probiotics) to combat the pathogenic biofilm-residing microbials. Probiotics were defined by the Food and
Agriculture Organization of the United Nations (FAO) and World Health Organization (WHO) as "live microorganisms, which when administered in adequate amounts, confer a health benefit on the host" [16]. Some studies already proposed a supportive role of probiotics in wound healing, e.g. of burns wounds infected with Pseudomonas aeruginosa in mice and rabbits $[17,18]$. Recent studies postulated chronic wound pathology may be a result of a dysbiosis of the skin microbiome [19]. Several hypotheses regarding the underlying mechanisms of the therapeutic effects were discussed. Pathogenic microbials can be repressed by natural selection in the competition for micronutrients and a modulation of the host immune system by probiotic bacteria is likely an important factor for the effectiveness of bacteriotherapy [20]. Additionally, some commensals from the naturally occurring human microbiome produce antimicrobial and bactericide substances [21-23]. Nevertheless, knowledge rendering targeted, secure application of bacteriotherapy, to treat human biofilm-challenged chronic wounds is still inaccurate and must be faced in further studies.

In this study, a novel human plasma biofilm model was used to mimic a biofilm-infected human wound environment, to analyse the efficiency of bacteriotherapy regarding the disruption of the EPS and elimination of biofilm-growing pathogenic microorganisms. Five clinically relevant pathogens Staphylococcus aureus, Pseudomonas aeruginosa, Staphylococcus epidermidis, Enterococcus faecium and Candida albicans were challenged to the lactic-acid producing probiotics Lactobacillus plantarum and Bifidobacterium lactis and to the human commensal Saccharomyces cerevisiae.

\section{Methods}

\section{Bacteria strains}

All strains were obtained from the Leibniz-Institute DSMZ-German Collection of Microorganisms and Cell Cultures. Details are given in Table 1.

\section{Experimental setup}

Human plasma biofilm model preparation

Plasma preserves and buffy coats from anonymous donors were obtained from the DRK-Blutspendedienst West (Hagen, Germany) and biofilm model were constructed as described previously [24]. In brief, residual erythrocytes in the buffy coat were removed by a centrifugation for $30 \mathrm{~min}$ at $3000 \mathrm{rpm}$ at room temperature (RT). The plasma preserve and the buffy coat were fused and collected in a sterile glass bottle. The content of the bottle was gently mixed and continuously shaked at $22{ }^{\circ} \mathrm{C}$.

A single hpBIOM was comprised of $1.5 \mathrm{ml}$ plasma solution. $1^{*} 10^{6} \mathrm{cfu} / 1.5 \mathrm{ml}$ pathogens were supplemented. 
Table 1 Applied pathogenic and probiotic bacteria

\begin{tabular}{|c|c|c|}
\hline Strain & DSM no & $\begin{array}{l}\text { Culture condition: } \\
\text { medium, } \\
\text { temperature }\end{array}$ \\
\hline Staphylococcus aureus subsp. aureus & 799 & $\mathrm{CSB}, 37^{\circ} \mathrm{C}$ \\
\hline Staphylococcus epidermidids & 20,044 & $\mathrm{CSB}, 37^{\circ} \mathrm{C}$ \\
\hline Pseudomonas aeruginosa & 939 & $\mathrm{CSB}, 37^{\circ} \mathrm{C}$ \\
\hline Enterococcus faecium & 2146 & $\mathrm{CSB}, 37^{\circ} \mathrm{C}$ \\
\hline Candida albicans & 1386 & $\mathrm{MEB}, 37^{\circ} \mathrm{C}$ \\
\hline $\begin{array}{l}\text { Lactobacillus plantarum subsp. plan- } \\
\text { tarum }\end{array}$ & 2601 & $\mathrm{MRS}, 37^{\circ} \mathrm{C}$ \\
\hline Bifidobacterium animalis subsp. lactis & 10,140 & $\mathrm{CSB}, 37^{\circ} \mathrm{C}$ \\
\hline Saccharomyces cerevisiae & 70,449 & MEB, $37^{\circ} \mathrm{C}$ \\
\hline
\end{tabular}

$18.26 \mu \mathrm{laCl}_{2}(500 \mathrm{mM})$ per $\mathrm{ml}$ plasma were applied, gently mixed and quickly transferred into wells of 12-well culture plates (Sarstedt AG \& Co., Nürnbrecht, Germany). The plates were incubated for $1 \mathrm{~h}$ on a rotation shaker at $50 \mathrm{rpm}$ and $37^{\circ} \mathrm{C}$. During this time, the plasma polymerized and a stable biofilm disc/clot with integrated pathogens was generated and could be used for further analyzes.

\section{Administration of probiotics}

L. plantarum, B. lactis and S. cerevisiae were grown in MRS, CSB or MEB medium for 2 days at $37^{\circ} \mathrm{C}$ with shaking at $50-100 \mathrm{rpm}$. The probiotics were diluted to provide $1^{*} 10^{9} \mathrm{cfu}$ in a maximum volume of $100 \mu \mathrm{l}$ medium. This concentration was applied on top of the biofilms, followed by an additional dose of $1^{*} 10^{9} \mathrm{cfu}$ after $2 \mathrm{~h}$ of incubation. Each pathogen was challenged to one single probiotic strain.

\section{Dissolving of the biofilm and quantification of the bacterial growth}

$2 \mathrm{~h}$ or $24 \mathrm{~h}$ after the second application of probiotics, the biofilm models were dissolved by the incubation with $1.5 \mathrm{ml}(1: 1 \mathrm{v} / \mathrm{v}) 10 \%(\mathrm{w} / \mathrm{v})$ bromelain solution (BromelainPOS $^{\circledR}$, RSAPHARM Arzneimittel GmbH, Saarbrücken, Germany) in $100 \mathrm{ml}$ phosphate buffered saline (PBS). By using a pipette tip, the discs were detached off the well margins and subsequently punctured to make the models more permeable for the enzymatic digestion. After $2 \mathrm{~h}$, the biofilm models were completely dissolved. For the quantification of the potentially survived pathogenic bacteria, $100 \mu \mathrm{l}$ aliquots from different dilution preparations were streaked out on CSA, MEA or MRS agar plates. The bacterial burden $(\mathrm{cfu} / \mathrm{ml})$ was determined by counting colonies with a Colony Counter Pen (eCount ${ }^{\mathrm{TM}}$, VWR Leicestershire, UK) after incubation over night at $37^{\circ} \mathrm{C}$.

\section{Scanning electron microscopy (SEM)}

Scanning electron microscopy (SEM) was used to analyse the bacterial morphology. The coagula were fixed with $0.1 \mathrm{M}$ cacodylate buffer containing $2.5 \%$ glutaraldehyde, $2 \%$ polyvinylpyrrolidone and $75 \mathrm{mM} \mathrm{NaNO}_{2}$ for $1 \mathrm{~h}$ at $4{ }^{\circ} \mathrm{C}$. Samples were washed in $0.1 \mathrm{M}$ cacodylate buffer without glutaraldehyde and subsequently incubated in a solution containing $2 \%$ arginine- $\mathrm{HCl}$, glycine, sucrose and sodium glutamate for $18 \mathrm{~h}$ at RT. The specimens were rinsed in distilled water followed by immersion in a mixture of each $2 \%$ tannic acid and guanidine- $\mathrm{HCl}$ for $5.5 \mathrm{~h}$ at RT. The samples were rinsed again in distilled water and incubated in a $1 \% \mathrm{OsO}_{4}$ solution for $30 \mathrm{~min}$ at RT. After three rinsing steps with distilled water the specimens were dehydrated, dried in liquid $\mathrm{CO}_{2}$, sputtered with gold palladium and finally examined with a Zeiss Sigma SEM (Zeiss, Oberkochen, Germany) using $2 \mathrm{kV}$ acceleration voltage and an inlens detector.

\section{Statistical analysis}

The experiments were performed in triplicates per donor for each pathogen/probiotic strain combination. Experimental data were analyzed by the statistic package GraphPadPrism 6 (GraphPad Software, Inc., La Jolla, USA). Data are presented as means \pm standard deviation (SD). Statistical analysis was performed by applying two-way ANOVA, followed by Tukey's HSD test as posthoc evaluation of multiple comparisons. A p-value of $p \leq 0.05$ was considered significant. (" $\mathrm{p} \leq 0.05$; *** $\mathrm{p} \leq 0.01$; $\left.{ }_{* * *: *}^{*} \mathrm{p} \leq 0.001\right)$.

\section{Results \\ Reproduction of the novel human plasma biofilm model (hpBIOM)}

The hPBIOM was produced by fusion of human plasma and the corresponding buffy coat from the same donor. After the addition of the bacteria and activation of the coagulation cascade, stable coagula-like biofilm discs with integrated pathogens were generated (Fig. 1a). By means of scanning electron microscopy, bacterial colonies were detected on the fibrin scaffold (Fig. 1b). Staining of the glycokalyx revealed the development of the EPS after $1 \mathrm{~h}$.

Interference of probiotic bacteria with pathogenic species This study involved a systematic analysis of the antimicrobial activity of three probiotics $L$. plantarum, B. lactis or $S$. cerevisiae against five clinically relevant pathogens P. aeruginosa, S. aureus, S. epidermidis, E. faecium and C. albicans. Lactobacillus plantarum eliminated the Pseudomonas infection after $4 \mathrm{~h}$ of incubation, except for biofilms from donor 1 and 2 (Fig. 2a). Finally, after $24 \mathrm{~h}$ 

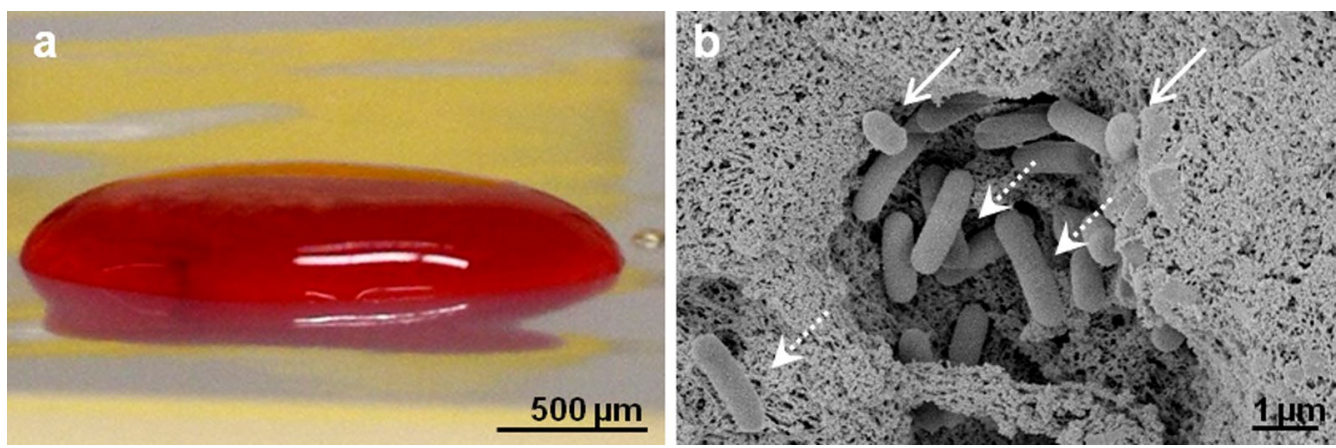

Fig. 1 Human plasma biofilm model (hpBIOM). a Stable coagula-like disc were generated. b By means of SEM, integrated microorganisms attached to the fibrin surface were visible

$P$. aeruginosa was successfully eradicated by $L$. plantarum in hpBIOMs from all donors. No recurrence of the pathogen was detected after $24 \mathrm{~h}$ in all plasma probes. The growth of $S$. aureus was also significantly affected in all hpBIOMs by $L$. plantarum, especially after $4 \mathrm{~h}$ (Fig. 2b). A $\log _{10}$ reduction rate between $0.9-2.1 \mathrm{cfu} / \mathrm{ml}$ was detected. In biofilms of plasma from donor 1 and 4 , the effect was negated after $24 \mathrm{~h}$. The influence of $L$. plantarum on the growth of S. epidermidis displayed variances between the individual donors (Fig. 2c). On the one hand no alteration was observed in hpBIOMs from donor 1 and 3, but, on the other hand, a slight reduction of pathogens was quantified in biofilms from donor 2. The application of L. plantarum on biofilms of E. faecium resulted in significant inhibition of bacterial growth with a reduction of $>1.8 \log _{10}$ phases. In contrast to the antibacterial effect of L. plantarum, no relevant antifungal response was detected towards C. albicans (Fig. 2e). $B$. lactis exerted a pathogen-reducing capacity towards $P$. aeruginosa as well as $E$. faecium, while the influence on $E$. faecium growth was strongly donor-specific (Fig. 3a, d). The growth rates of $S$. aureus, S. epidermidis and C. albicans showed no differences between $B$. lactis treated and non-treated conditions after $4 \mathrm{~h}$ of incubation (Fig. 3b, c, e). The application of the yeast $S$. cerevisiae resulted into moderate but significant reduction of the pathogens $S$. aureus and S. epidermidis (Fig. 4b, c). The antimicrobial efficiency towards Pseudomonas varied in the biofilms. Inhibitory as well as slightly growth-promoting effects were detected (Fig. 4a).

\section{Combat between P. aeruginosa and L. plantarum:} a presentation via scanning electron microscopy (SEM)

SEM analysis should give more insight into the organization of L. plantarum while eliminating Pseudomonas (Fig. 5). During the experiments, L. plantarum was applied on top of the biofilm. The eradication process was documented after $1 \mathrm{~h}$ and $4 \mathrm{~h}$ of incubation. The SEM micrographs illustrated, that L. plantarum moved into the hpBIOM and arrived at the Pseudomonas colony after $1 \mathrm{~h}$ (Fig. 5a arrow, straight lines). The number of Lactobacilli increased with time. Scattered probioticpathogen interactions were visible (Fig. 5a). L. plantarum produced a complex glycokalyx, more rapidly compared to Pseudomonas (Fig. 5a, b). This matrix seemed to coat the pathogen, finally, leading to the death of the bacteria (Fig. 5c).

\section{Discussion}

In Europe, at least 5 million people suffer from leg ulcer, as a representative chronic wound. Approximately, 4 million diabetic patients will develop leg or food ulcer within the next 10 years [2]. Patients and their relatives have to bear great misery and the economic load for the health care system is enormous [25].

Non-healing wounds can be considered as a variant of chronic infection. Endogenously, the wound healing process stocks in the inflammatory phase and, externally, chronic wounds are often infected with bacteria and fungi, residing within a self-constructed biomatrix [26]. These biofilms delay wound healing and, due to tremendous survival strategies, are difficult to eliminate.

Various in-vitro biofilm models have been developed for research. The majority consist of bacteria, attached on any adhesive surface. All lack characteristics of the human wound environment and the immune competence. This study used an innovative human plasma biofilm model (hpBIOM), which perfectly mimics a biofilm-challenged human wound milieu. During the initial phases of wound healing, homeostasis and inflammation, vascular permeability increases and blood plasma diffuses into the damaged tissue [27]. Amongst others, the plasma dilutes toxic degradation products, to physically cleaning the wounded area. Further advantage of 

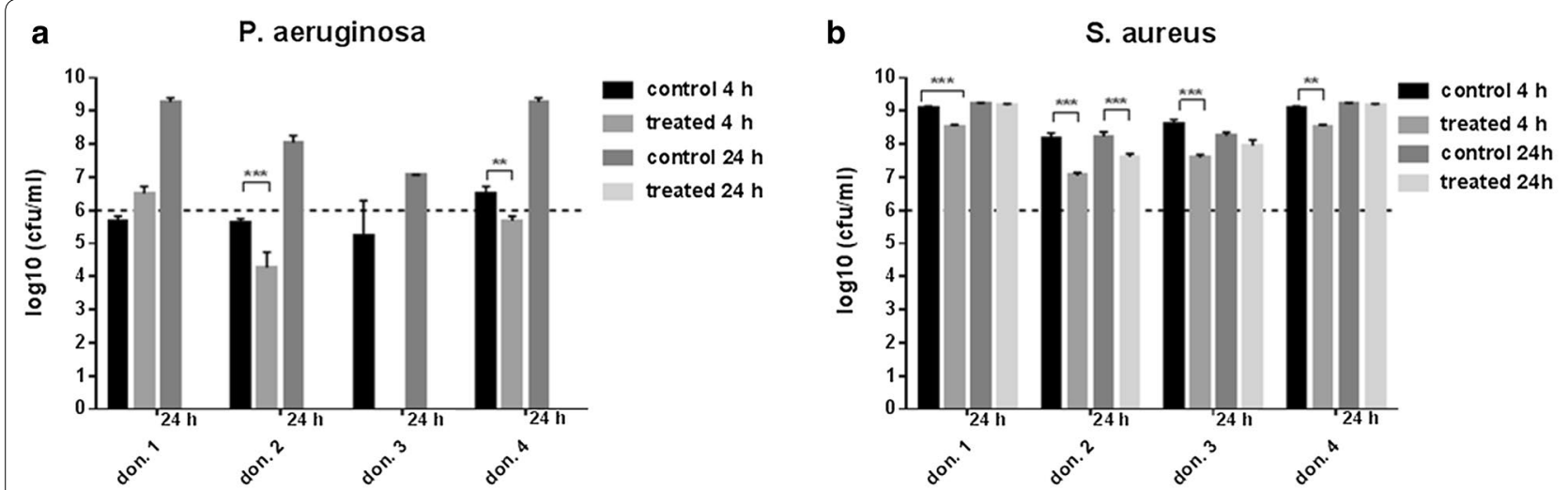

\section{S. epidermidis}
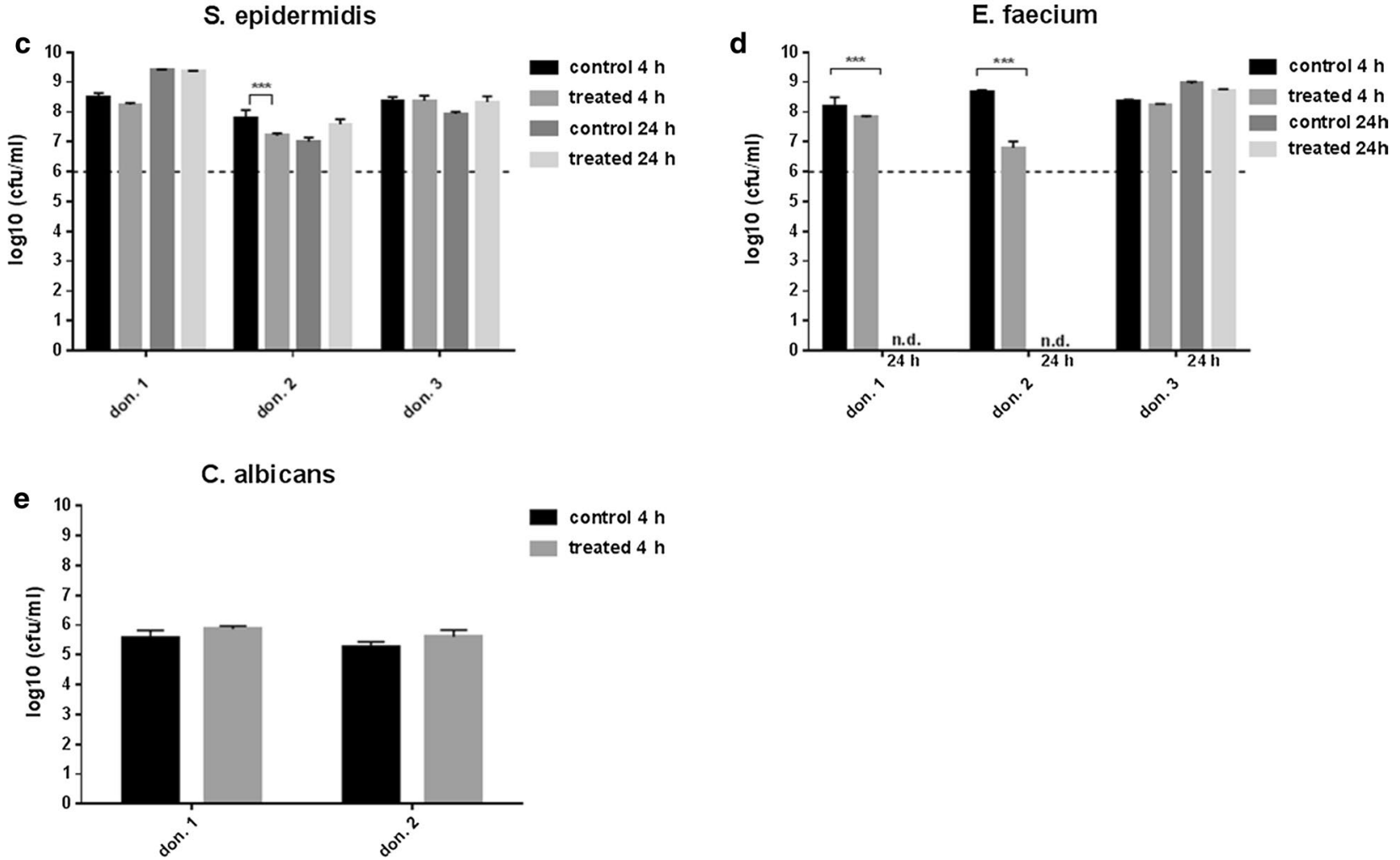

Fig. 2 Influence of L. plantarum on microbial growth in biofilms. a With the exception of two donors, L. plantarum successfully eliminated the P. aeruginosa infection after $4 \mathrm{~h}$ of incubation. After $24 \mathrm{~h}$, P. aeruginosa was finally destroyed in biofilms from all donors. b-d The pathogens $S$. aureus, S. epidermidis and E. faecium also displayed a slight growth inhibition. The effects are dependent on the individual plasma. e The growth rate of C. albicans showed no inhibitory influence of $L$. plantarum. don., donor; nd, not determined. All experiments were performed in triplet per donor $\left({ }^{*} p \leq 0.05 ;{ }^{* *} p \leq 0.01 ;{ }^{* * *} p \leq 0.001\right)$

using human plasma, was the availability of the immune competence for fighting the infection. It contains lymphocytes, granulocytes and monocytes-immune cells that protect the system from infection by microorganisms and decompose damaged cells, resulting in an additional, physiological cleaning effect $[28,29]$. The platelets and the complement system in the plasma were utilized to produce coagula-like stable discs, after the administration of pathogenic microorganisms (Fig. 1). Scanning electron microscopy images of $P$. aeruginosa biofilms, stained to the glycokalyx, demonstrated, that the pathogens (as well as probiotics) attached to the fibrin matrix, generate microcolonies and produce EPS (Figs. 1b, 5a-c). Due to all these properties the hpBIOM is postulated to be an appropriate in-vitro biofilm model for translational approach to the clinical situation. 


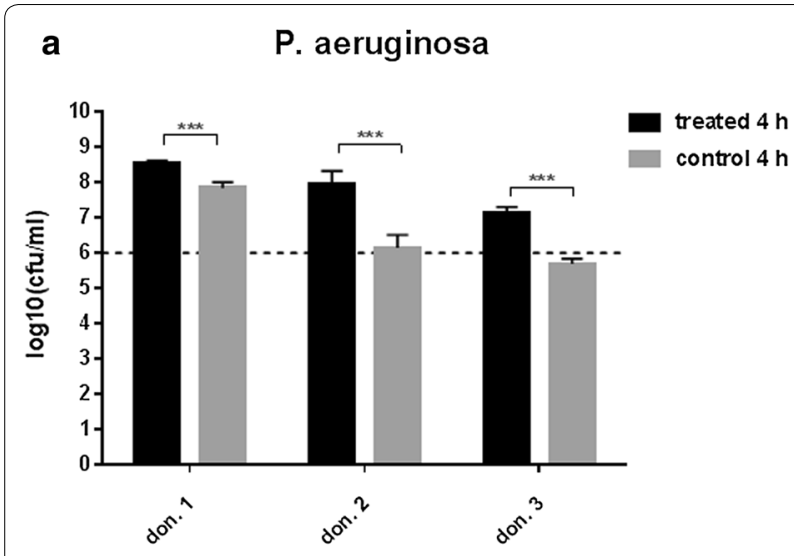

b S. aureus
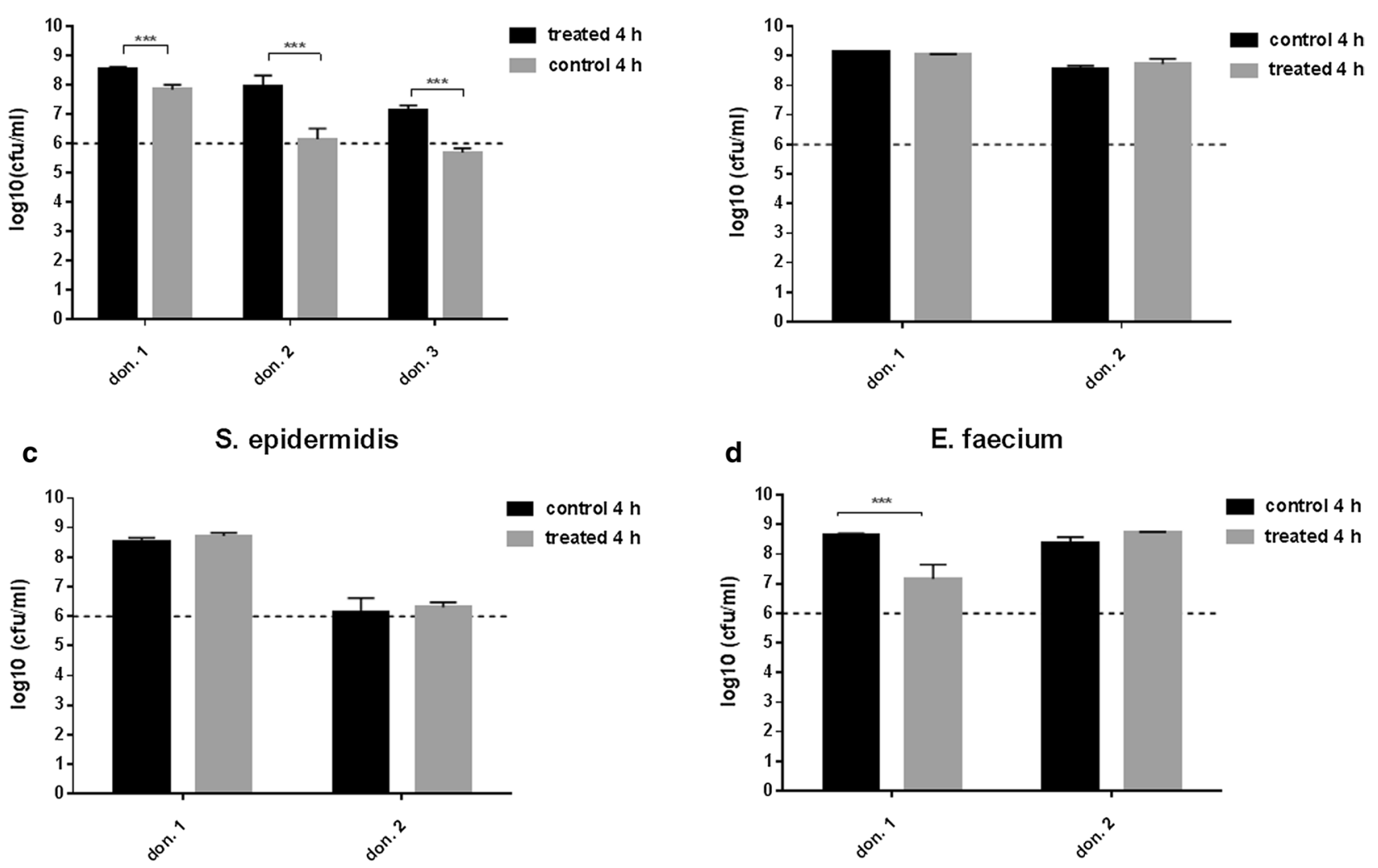

e

C. albicans

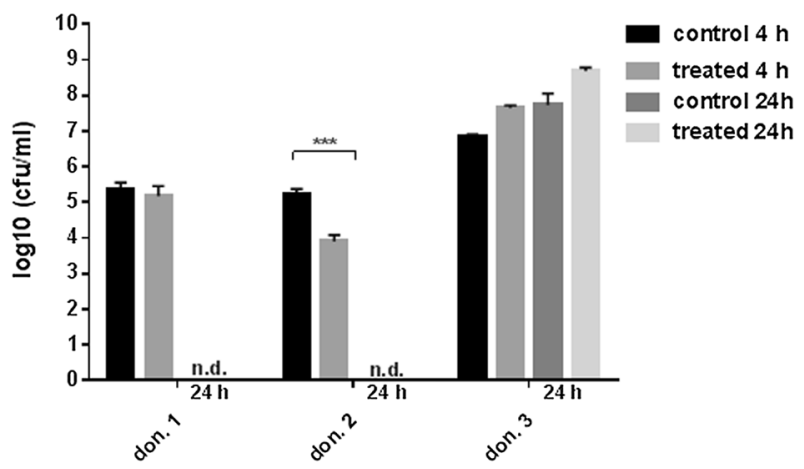

Fig. 3 Antimicrobial activity of B. lactis in the hPBIOM. b, c B. lactis exerted no inhibitory effects on S. aureus and S. epidermidis. a, d, e Growth-reducing capacity was determined towards Pseudomonas, E. faecium and in one donor towards C. albicans up to $2 \log _{10}$-reduction rates. The effect was strongly plasma-dependent. don., donor; nd, not determined. All experiments were performed in triplet per donor $\left({ }^{*} p \leq 0.05 ;{ }^{* *} p \leq 0.01\right.$; $* * * 0 \leq 0.001)$

\section{Bacteriotherapy for eliminating pathogenic microorganisms in biofilms}

Health-promoting effects of "good " lactic acid-producing bacteria were already described centuries ago, especially those belonging to the species Bifidobacterium and Lactobacillus, by inhibiting the growth of pathogenic bacteria within the colon. Different probiotics are already in use to treat dysbiosis and infections of the gastrointestinal- and urinary tract and dental diseases, e.g. pouchitis [7, 16, 30, 31].

Many studies propose better outcomes after bacteriotherapy by using L. plantarum, e.g. in animal models of $P$. aeruginosa infected burn wounds or chronic wounds in diabetic mice. Even a topically applied prophylactic administration of $L$. plantarum induced a health benefit $[17,30,32]$. Some in-vitro studies using surface-attached 


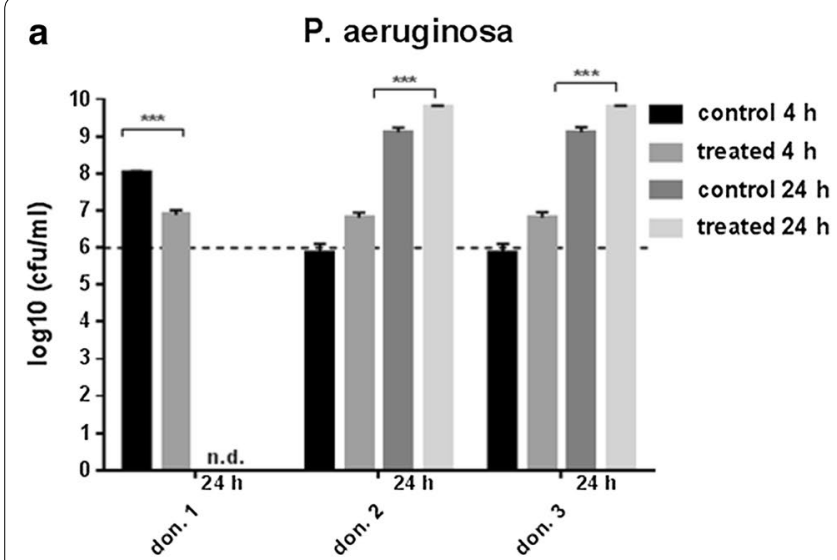

b

S. aureus

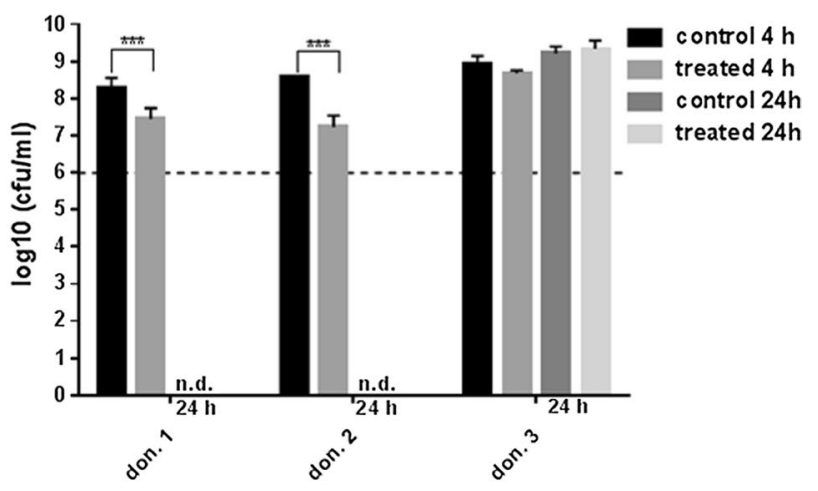

\section{S. epidermidis}
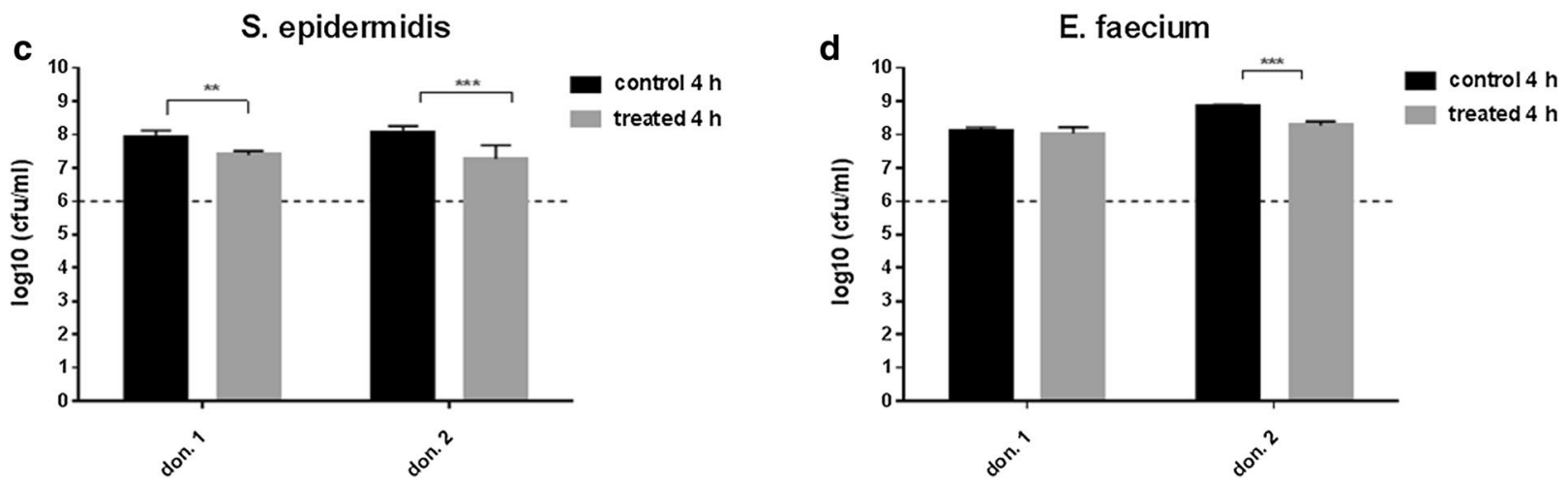

Fig. 4 Effect of S. cerevisiae on the survival of pathogens in the hpBIOM. a, b Dependent on the donor, S. cerevisiae showed growth-inhibiting capacities towards P. aeruginosa and S. aureus. c, d S. epidermidis and E. faecium were not influenced in a clinically relevant concentration. don., donor; nd, not determined. All experiments were performed in triplet per donor $\left({ }^{*} p \leq 0.05 ;{ }^{* *} p \leq 0.01 ;{ }^{* * *} p \leq 0.001\right)$
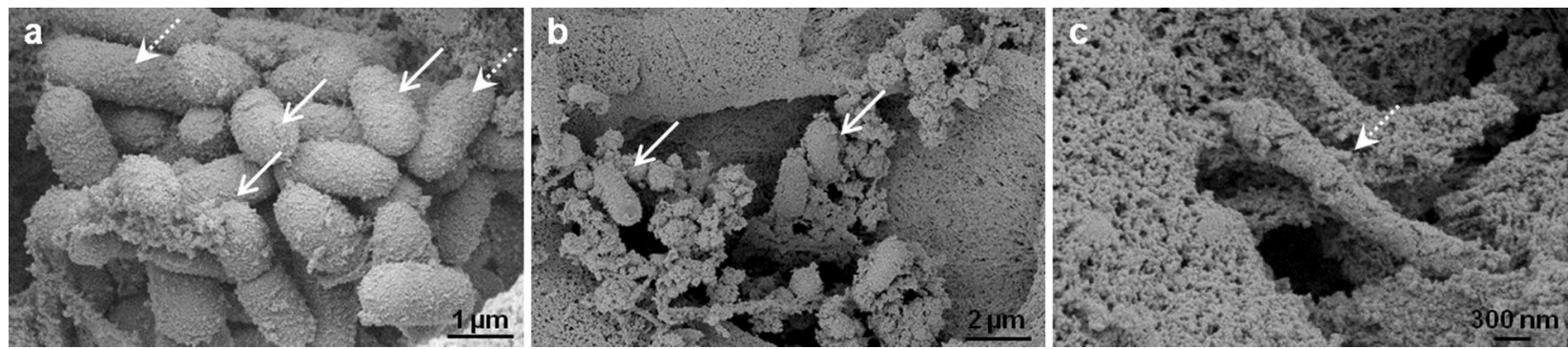

Fig. 5 Scanning electron microscopy (SEM) images of a P. aeruginosa hpBIOMs. a Within the biofilm, bacteria were attached in close vicinity on the surface of the matrix, to develop microcolonies (arrows, dotted lines). $\mathbf{b}$ One $\mathrm{h}$ after supplementation of L. plantarum, probiotics arrive the colonies (arrows, straight lines), production of the EPS and scattered interactions were visible. c After $4 \mathrm{~h}$, L. plantarum has produced a strong glycokalyx, which seemed to cover and subsequently destroy Pseudomonas

biofilms, challenged the pathogens to different types of living lactic acid-producing bacteria as well as supernatants or isolated proteins, and confirmed the antimicrobial activity and healing-promoting effects [33-39]. The success was dependent on the applied pathogens and probiotics and their concentrations. However, there is a great need for research addressing the potential of bacteriotherapy and the understanding of the mechanisms in more detail. This study transferred the investigation to the newly established human plasma biofilm model. The selection of pathogenic bacteria was based on the WHO list of priority pathogens for R\&D of new antibiotics published in February 2017 [15]. Additionally, a fungal contamination with C. albicans was examined. 
Plasma preserves from different donors were used for the investigation. The results were not pooled, due to the different immune competences of the donors and the potential influence on the antimicrobial efficiency. In the hpBIOM, it was possible to demonstrate and to confirm the enormous antimicrobial efficiency of L. plantarum towards Pseudomonas infections (Fig. 2a). By means of SEM, it was possible to visualize the migration into the biofilm and direct pathogen-probiotic interaction (Fig. 5a, b). Furthermore, L. plantarum extensively produced a glycokalyx, which seemed to cover and destroy Pseudomonas (Fig. 5c). Supplementation of L. plantarum to $S$. aureus, S. epidermidis and E. faecium also induced slight but significant growth reductions (Fig. 2b-d), which was not shown before. The exact mechanisms resulting in the reduction or elimination of these bacteria is currently under investigation in this system. Different possibilities are postulated in other publications. For instance, different lactobacilli species have antielastase activity against $P$. aeruginosa [33]. Additionally, the effects of $L$. plantarum were assigned to the secretion of antimicrobial substances, like 4,5-dihydroxy-2,3-pentanedione and 2-methyl-2,3,3,4-tetrahydroxytertahydrofurane, which inhibits quorum sensing [38]. Other antimicrobial substances like hydrogen peroxide, benzoic acid or lactic acid are also secreted by $L$. plantarum [36]. The effect was donor- and time-specific, and thereby considered to be dependent on the immune system of the donor. This thesis was already proved in the gut, where different Bifidobacteria as well as Lactobacilli exerted stimulatory effect on the immune system [16]. This has to be evaluated in progressive studies. Additionally, the constitution of the bacterial cell membrane seems to be a limiting factor, because the highest growth-reducing effects were detected against gram-negative bacteria. The growth rate of $C$. albicans was not affected (Fig. 2e). This species is also surrounded by a strong cell wall. Interestingly, B. lactis also exerted a reducing activity towards Pseudomonas and E. faecium (Fig. 3a, d) and even the yeast $S$. cerevisiae showed slight but significant inhibitory effects on S. aureus, S. epidermidis and E. faecium (Fig. $4 \mathrm{~b}-\mathrm{d}$ ). These capacities were not yet determined in human biofilms. Although the reduction of the bacterial burden seemed not to be tremendous in some combinations, it can have major relevance for the wound therapy, because it enhances the chance of reducing bacterial load by the individual immune system. Further tests with a higher number of probiotics or their combinations will be performed, to examine, whether this will improve antimicrobial outcome.

Summarized, this study successfully reproduced a novel human biofilm model. This system still represents an invitro model and bares limitations like a time-limited stability or the lack of skin cells. Nevertheless, several improvements were developed compared to current biofilm models. It involves essential factors for analysing biofilms in a translational research approach, namely the individual immune competence and a human wound environment. By means of the hpBIOM, it was possible to systematically screen growth-reducing activity of three probiotics towards five clinically relevant pathogens. It was possible to visualize the elimination process of $L$. plantarum against $P$. aeruginosa. Finally, additional insights into the influence of the probiotic microorganisms $B$. lactis and S. cerevisiae could be efficiently obtained. These effects are described for this study design and could be differ after using other concentrations of probiotics or pathogens, respectively. In future studies, the investigation of bacteriotherapy by means of the hpBIOM should be expanded with regard to subcellular and molecular insights. Additionally, the portfolio of probiotics should be increased and in particular, combined therapies of $L$. plantarum and other effective probiotics should be investigated using the hPBIOM.

\section{Conclusions}

A novel human biofilm model, which includes two essential factors for the analysis of biofilms in a translational approach, namely, a human wound milieu and individual human immune competence was reproduced. Especially, the probiotic $L$. plantarum is able to eliminate $P$. aeruginosa and differentially inhibits the growth of the tested pathogens, except of C. albicans. B. lactis and S. cerevisiae exert growth-inhibiting influence. The antimicrobial activity was strikingly donor-specific.

\section{Explanation and importance for the field}

The presence of biofilms impairs wound healing and reflects one pivotal factor in the development of chronic wounds. Biofilm-growing bacteria display increased antibiotic resistances: Knowledge of the biofilm environment, behavior of the bacteria and novel therapeutic options are prerequisite for improving wound management. Appropriate model systems for the analyses in a direct translational approach are still missing. They lack the human wound milieu and the immune competence. This study used a novel human plasma biofilm model and provides further insights into the efficiency of bacteriotherapy by means of probiotics.

\footnotetext{
Abbreviations

B.: Bifidobacterium; C.: Candida; CSB: Casein-Soy-Bouillon; cfu: colony-formingunit; don.: donor; E.: Enterococcus; EPS: extracellular polymeric substance; Fig.: figure; h: hour; hpBIOM: human plasma biofilm model; L.: Lactobacillus; MEB: Malt-Extract-Bouillon; Min: minute; MRS: Man-Rogosa-Sharpe; P.: Pseudomonas; rpm: round per minute; SD: standard deviation; SEM: scanning electron microscopy.
} 


\section{Acknowledgements}

We thank Verena Koester and Susanne Haußmann for technical assistance.

\section{Authors' contributions}

$M B$ and EKS designed the study. MB and JT carried out the experiments; EN and WA supported the SEM; LB discussed the manuscript, MB analyzed results, performed statistical analysis and made the figures; $\mathrm{MB}$ and EKS drafted the manuscript. All authors read and approved the final manuscript.

\section{Funding}

This project was funded by the Interne Forschungsfoerderung UW/H (IFF2018-61)

\section{Availability of data and materials}

All data and material are available.

\section{Ethics approval and consent to participate}

Not required.

\section{Consent for publication}

All authors give consent for publication.

\section{Competing interests}

The authors declare that they have no competing interests.

\section{Author details}

1 Institute of Translational Wound Research, Centre for Biomedical Education and Research (ZBAF), Witten/Herdecke University, Stockumer Street 10, 58453 Witten, Germany. ${ }^{2}$ Institute for Medical Laboratory Diagnostics, Centre for Clinical and Translational Research (CCTR), HELIOS University Hospital Wuppertal, Wuppertal, Germany. ${ }^{3}$ Department of Biological and Material Sciences in Dentistry, School of Dentistry, Faculty of Health, Witten/Herdecke University, Witten, Germany. ${ }^{4}$ Institute for Health Care Research in Dermatology and Nursing, University Medical Center Hamburg-Eppendorf (UKE), Hamburg, Germany.

Received: 11 April 2019 Accepted: 17 July 2019

Published online: 25 July 2019

\section{References}

1. Dissemond J, et al. Diagnosis and treatment of chronic wounds: current standards of Germany's Initiative for Chronic Wounds e V. J Wound Care. 2017;26(12):727-32.

2. Krech T, Traber JÜ. Bakterien in chronischen Wunden. Ars Medici. 2010;23:960-4.

3. Siddiqui AR, Bernstein JM. Chronic wound infection: facts and controversies. Clin Dermatol. 2010;28(5):519-26.

4. James GA, et al. Biofilms in chronic wounds. Wound Repair Regen. 2008;16(1):37-44.

5. Costerton JW. Introduction to biofilm. Int J Antimicrob Agents. 1999;11(34):217-21 (discussion 237-9).

6. Costerton JW, Stewart PS, Greenberg EP. Bacterial biofilms: a common cause of persistent infections. Science. 1999:284(5418):1318-22.

7. Zhao G, et al. Biofilms and inflammation in chronic wounds. Adv Wound Care (New Rochelle). 2013;2(7):389-99.

8. Spiliopoulou Al, et al. Bacterial adhesion, intracellular survival and cytokine induction upon stimulation of mononuclear cells with planktonic or biofilm phase Staphylococcus epidermidis. FEMS Microbiol Lett. 2012;330(1):56-655.

9. Tzaneva $V$, et al. Antibiotic treatment and resistance in chronic wounds of vascular origin. Clujul Med. 2016;89(3):365-70.

10. Costerton JW, Stewart PS. Battling biofilms. Sci Am. 2001;285(1):74-81.

11. Stewart PS, Costerton JW. Antibiotic resistance of bacteria in biofilms. Lancet. 2001;358(9276):135-8.

12. Stewart PS, et al. Biofilm penetration and disinfection efficacy of alkaline hypochlorite and chlorosulfamates. J Appl Microbiol. 2001;91(3):525-32.

13. Schultz GS, et al. Wound bed preparation: a systematic approach to wound management. Wound Repair Regen. 2003;11(Suppl 1):1-28.

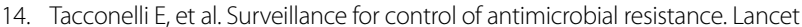
Infect Dis. 2018;18(3):e99-e106.

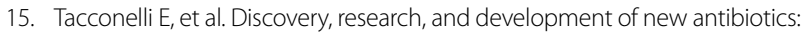
the $\mathrm{WHO}$ priority list of antibiotic-resistant bacteria and tuberculosis. Lancet Infect Dis. 2018;18(3):318-27.

16. Vuotto C, Longo F, Donelli G. Probiotics to counteract biofilm-associated infections: promising and conflicting data. Int J Oral Sci. 2014;6(4):189-94.

17. Argenta A, et al. Local application of probiotic bacteria prophylaxes against sepsis and death resulting from burn wound infection. PLoS ONE. 2016;11(10):e0165294

18. Satish L, et al. Local probiotic therapy with Lactobacillus plantarum mitigates scar formation in rabbits after burn injury and infection. Surg Infect (Larchmt). 2017;18(2):119-27.

19. Lukic J, et al. Probiotics or pro-healers: the role of beneficial bacteria in tissue repair. Wound Repair Regen. 2017;25(6):912-22.

20. Sonal Sekhar M, et al. Topical application/formulation of probiotics: will it be a novel treatment approach for diabetic foot ulcer? Med Hypotheses. 2014;82(1):86-8

21. Zipperer $A$, et al. Human commensals producing a novel antibiotic impair pathogen colonization. Nature. 2016:535(7613):511-6.

22. Francolini I, et al. Antifouling polyurethanes to fight device-related staphy lococcal infections: synthesis, characterization, and antibiofilm efficacy. Pathog Dis. 2014;70(3):401-7.

23. Francolini I, et al. Antifouling and antimicrobial biomaterials: an overview. APMIS. 2017;125(4):392-417.

24. Besser M, Stuermer EK. Efficiency of antiseptics in a novel 3-dimensional human plasma biofilm model (hpBIOM). NPJ Biofilms and Microbiomes. 2019

25. Purwins S, et al. Cost-of-illness of chronic leg ulcers in Germany. Int Wound J. 2010;7(2):97-102.

26. Misic AM, Gardner SE, Grice EA. The wound microbiome: modern approaches to examining the role of microorganisms in impaired chronic wound healing. Adv Wound Care (New Rochelle). 2014;3(7):502-10.

27. Velnar T, Bailey T, Smrkolj V. The wound healing process: an overview of the cellular and molecular mechanisms. J Int Med Res. 2009;37(5):1528-42.

28. Demidova-Rice TN, Hamblin MR, Herman IM. Acute and impaired wound healing: pathophysiology and current methods for drug delivery, part 1: normal and chronic wounds: biology, causes, and approaches to care. Adv Skin Wound Care. 2012;25(7):304-14.

29. Gurtner GC, et al. Wound repair and regeneration. Nature. 2008;453(7193):314-21.

30. Baquerizo Nole, K.L., E. Yim, J.E. Keri, Probiotics and prebiotics in dermatology. J Am Acad Dermatol, 2014; 71(4): 814-21.

31. Comelli EM, et al. Selection of dairy bacterial strains as probiotics for oral health. Eur J Oral Sci. 2002;110(3):218-24.

32. Dai T, et al. Topical antimicrobials for burn wound infections. Recent Pat Antiinfect Drug Discov. 2010;5(2):124-51.

33. Alexandre Y, et al. Screening of Lactobacillus spp. for the prevention of Pseudomonas aeruginosa pulmonary infections. BMC Microbiol. 2014;14:107.

34. Butler $\mathrm{E}$, et al. A pilot study investigating lactic acid bacterial symbionts from the honeybee in inhibiting human chronic wound pathogens. Int Wound J. 2016;13(5):729-37.

35. Valdez JC, et al. Interference of Lactobacillus plantarum with Pseudomonas aeruginosa in vitro and in infected burns: the potential use of probiotics in wound treatment. Clin Microbiol Infect. 2005;11(6):472-9.

36. Ramos AN, et al. Antipathogenic properties of Lactobacillus plantarum on Pseudomonas aeruginosa: the potential use of its supernatants in the treatment of infected chronic wounds. Wound Repair Regen. 2012;20(4):552-62.

37. Ramos AN, et al. Effect of Lactobacillus plantarum and Pseudomonas aeruginosa culture supernatants on polymorphonuclear damage and inflammatory response. Int Immunopharmacol. 2010;10(2):247-51.

38. Ramos AN, et al. Compounds from Lactobacillus plantarum culture supernatants with potential pro-healing and anti-pathogenic properties in skin chronic wounds. Pharm Biol. 2015:53(3):350-8.

39. Tsiouris CG, et al. The efficacy of probiotics as pharmacological treatment of cutaneous wounds: meta-analysis of animal studies. Eur J Pharm Sci. 2017;104:230-9.

\section{Publisher's Note}

Springer Nature remains neutral with regard to jurisdictional claims in published maps and institutional affiliations. 